\title{
Nash Equilibrium of an Energy Saving Strategy with Dual Rate Transmission in Wireless Regional Area Network
}

\author{
Zhanqiang Huo, ${ }^{1}$ Xiaowei Li, ${ }^{1}$ Shunfu Jin, ${ }^{2}$ and Zhiheng Wang' \\ ${ }^{1}$ College of Computer Science and Technology, Henan Polytechnic University, Jiaozuo, Henan 454000, China \\ ${ }^{2}$ College of Information Science and Engineering, Yanshan University, Qinhuangdao, Hebei 066004, China \\ Correspondence should be addressed to Zhanqiang Huo; hzq@hpu.edu.cn
}

Received 26 July 2017; Revised 19 October 2017; Accepted 25 October 2017; Published 20 November 2017

Academic Editor: Donatella Darsena

Copyright (c) 2017 Zhanqiang Huo et al. This is an open access article distributed under the Creative Commons Attribution License, which permits unrestricted use, distribution, and reproduction in any medium, provided the original work is properly cited.

\begin{abstract}
Wireless regional area network (WRAN) adopts centralized network architecture and is currently one of the most typical cognitive radio networks. In order to reduce the energy consumption of the communication networks with the constraint of spectrum resource utilization, a working sleep mechanism is introduced into the base station (BS), and a novel energy saving strategy with dual rate transmission is proposed. Combining the multiple-vacation queue and priority queue, using the quasi-birth-death process and the matrix-geometric solution method, we assess the average latency and the forced termination probability of secondary user packets, as well as the energy saving ratio and the channel utilization of system. Based on the revenue-expenditure structure, a profit function is built, and then the Nash equilibrium behavior and the socially optimal behavior are investigated. With the help of the particle swarm optimization, an intelligent optimization algorithm to search the socially optimal arrival rate of secondary user packets is presented. In order to unify the arrival rates of secondary user packets with Nash equilibrium and social optimization, a reasonable pricing policy is formulated. In addition, system experiments are carried out to verify the effectiveness of the energy saving strategy and the rationality of the pricing policy.
\end{abstract}

\section{Introduction}

With the rapid development of wireless communication technology, wireless networks play an increasingly important role in various fields, and their users and services are explosive growth. According to reports $[1,2]$, the energy consumption caused by the information and communications technology infrastructure increases by about $16-20 \%$ annually and produces about $2 \%$ of the global $\mathrm{CO}_{2}$ emissions. Therefore, for solving the shortage of spectrum resources, wireless communication technology is also facing the challenge of reducing communication energy consumption. Energy saving strategy for cognitive wireless networks has become a research hotspot.

A cognitive radio system is one of promising solutions that can relieve the spectrum shortage problem by using spectrum overlay sharing [3]. IEEE 802.22 is the first formal standards based on cognitive radio technology; the goal is to establish air interface specification for wireless regional area network [4]. Balaji et al. proposed approaches for cooperative spectrum sensing using perceptron learning framework to meet the functional requirement of IEEE 802.22 WRAN standard [5]. Bagwari et al. proposed a reliable spectrum sensing scheme using dual detectors and double threshold, which improves detection performance and takes smaller detection time period than cyclostationary detection [6]. Joshi et al. studied the channel selection problems in IEEE 802.22 WRAN based on fuzzy-logic-based algorithm [7].

BSs in centralized network architecture widely use the sleep modes mechanism to achieve the purpose of reducing energy consumption [8]. Sleep mode is one of the most widely investigated techniques to decrease energy consumption in cellular access networks [9]. Considering the traffic dynamics in time domain and space domain, $\mathrm{Wu}$ et al. put forward a kind of pico BS sleep mode control method oriented heterogeneous wireless networks, which reduces the computational complexity and energy costs [10]. With different access technology of the next generation optical fiber access 
network, Lannoo et al. analyzed the impact of low energy mode (e.g., sleep mode) on the energy efficiency [11].

In recent years, some scholars apply the game theory in economics to cognitive wireless network spectrum allocation strategy [12]. Formulating the spectrum access procedure of second users as a noncooperative game, $[13,14]$ studied the spectrum access decision problem and [15] analyzed the game behavior of making decisions among multiple participants. In order to achieve maximal revenue and social welfare, Tran et al. studied a price-based spectrum access control in cognitive radio networks [16].

On the basis of the above research, this paper proposes a novel energy saving strategy with dual rate transmission for BS in IEEE 802.22 WRAN. The energy consumption of the BS is approximately in line with the central processing unit utilization, so the most direct method of conserving energy is to operate the BS at lower voltage and frequency. These will reduce the speed of the packets. BS may be in a state of low speed transmission during the working sleep period; that is, the secondary user packets are transmitted with a low speed and may also be in a state of no data transmission; that is, no data packets in the system need to be transmitted, so that the energy consumption can be conserved. BS will be in a state of high speed transmission during an awake period; the transmission speed of the primary user (PU) or secondary user (SU) packets will be higher to ensure the quality of services. The PU packets are granted high priority to occupy the spectrum for data transmission by charging the SU packets to occupy the spectrum in opportunistic approach. Integrating theories of multiple working vacations queue and priority queue, considering the number of cognitive user packets and state of BS, and using quasi-birth-death process and the matrix-geometric solution method in this paper, the system performance of the energy saving strategy is evaluated and the Nash equilibrium and socially optimal behavior of energy saving strategy are analyzed using game theory thought. Moreover, an intelligent optimization algorithm is presented to search the socially optimal arrival rate of SU packets based on the revenue-expenditure structure, and a reasonable pricing scheme is formulated to unify the individually and socially optimal arrival rates.

\section{Energy Saving Strategy Based on Dual Rate Transmission}

The IEEE 802.22 WRAN adopts centralized network architecture; BS and users equipment are the subject of network. Power management, spectrum allocation, and scheduling control are performed by the BS and user data packets access core network through the air interface of the BS. The PU data packet has a high priority and can preferentially occupy the spectrum. On the premise of ideal spectrum sensing, the arrival of SU packets does not affect the transmission of PU data packets. SU packets occupy the spectrum in opportunistic approach, and their transmissions may be interrupted by the arrival of the PU data packet. In the absence of data packets (PU packets or SU packets), the traditional IEEE 802.22 WRAN BS is still in a high speed state, which results in a waste of energy.

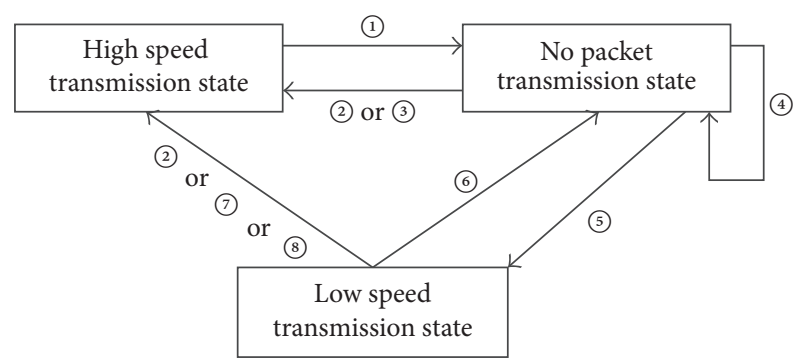

FIGURE 1: State transition of the BS.

This paper proposes an energy saving strategy based on dual rate transmission. The $\mathrm{BS}$ is in a high speed transmission state during an awake period, PU data packets have priority data transmission because of preemptive priority, and SU packets are opportunistic transmitted at a high rate in accordance with the first come first service (FCFS) rule. In order to reduce energy consumption, when there is no packet, the BS changes from an awake period to a working sleep period and starts a working sleep timer. For the response sensitive traffic, the sleeping parameter should be set higher; on the contrary, the sleeping parameter should be set lower. At the working sleep period, if there is no packet to be transmitted, the BS will turn to a fully sleep state. In a fully sleep state, the BS will listen to the spectrum at the beginning of each slot and determine whether the PU packet arrives. During working sleep period, if a PU packet arrives, the working sleep timer is terminated immediately, and the BS will return to high speed transmission state; the packets will be transmitted at a high rate. That is to say, PU packets are transmitted on one speed but not affected by the dual rate transmission mode.

At the awake period, all the packets in the system are being transmitted at high speed. Considering the energy saving effect of the system and the transmission quality of the SU packets, SU packets arriving at the working sleep period are transmitted at a low rate. When the packet is finished in the working sleep period, if there are SU packets in the system waiting transmission, the working sleep timer is stopped, and the BS enters an awake period from the working sleep period; otherwise, the BS stays in the working sleep period and goes into a no data transmission state. If the working sleep timer expires and there is no data packet to be transmitted in the system, the BS enters the next working sleep period and restarts the working sleep timer.

The conversion process among the BS states based on dual rate transmission energy saving strategy is shown in Figure 1.

The events in the conversion process among the BS states (high speed transmission, low speed transmission, and no data transmission) are described as follows:

(1) All the user packets in the system are transmitted at a high speed.

(2) A PU packet arrives.

(3) The working sleep timer expires with packets arriving at the system. 


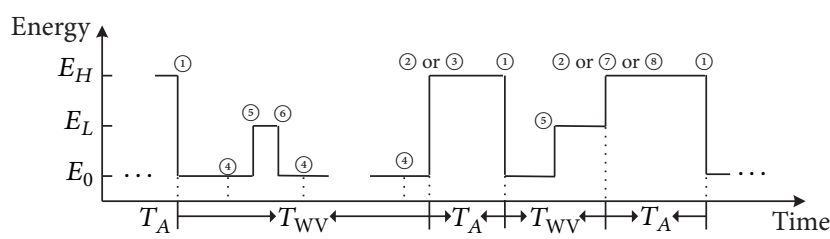

FIGURE 2: Timing changes of energy saving strategy with dual rate transmission.

(4) The working sleep timer expires with no packet arriving at the system.

(5) The working sleep timer does not expire but at least one SU packet arrives.

(6) The SU packet completes the transmission at a low rate, and no SU packets arrive during the transmission period.

(7) The working sleep timer expires, but the transmission of $\mathrm{SU}$ packet that is being transmitted at a low rate is not complete.

(8) SU packets are transmitted at a low rate, with SU packets arrive at the system.

Let $E_{H}, E_{L}$, and $E_{0}$ be the energy consumption per unit time when the BS is in a high speed transmission state, low speed transmission state, and no packet transmission state, respectively; we get $E_{H}>E_{L}>E_{0}$. Corresponding to the state transition in BS, the timing changes of energy saving strategy with dual rate transmission are shown in Figure 2.

In Figure 2, $T_{A}$ indicates an awake period. An awake period $T_{A}$ is a time duration from a BS entering a high speed transmission state to the time point that there is no data needed to be transmitted. A working sleep period $T_{\mathrm{WV}}$ is a period of time when a BS departs from a high speed transmission state and then returns to a high speed transmission state again. A working sleep period $T_{\mathrm{WV}}$ is often made up of one or more groups of no data transfer periods and low speed transmission periods. In the awake period, all air interface of the BS can transmit data packets. An awake period changes to a working sleep state by turning off some of the air interface of the BS.

Dual rate transmission energy saving strategy in IEEE 802.22 WRAN takes into account both the purpose of energy saving and the user's response requirements. The energy consumption of the BS in the working sleep period is lower than that in the awake period; meanwhile, some user data packets may be transmitted during the working sleep period.

\section{System Model and Model Analysis}

3.1. System Model. Considering the digital characteristics of modern communication network, a discrete time queuing model is established. Discrete time queuing theory divides the time axis into equal intervals, called slots, which is the smallest unit of transmission.

Based on the proposed energy saving strategy, the single channel case of the early-arrival system is considered. The arrival process of the PU packet and the arrival process of the SU packet are independent of each other, and the transmission time is independent of each other too.

Following an early-arrival system, we suppose that the arrival of data packets occurs at the beginning of a slot, marked as $\left(n, n^{+}\right)(n=1,2, \ldots)$, while the departure of data packets occurs at the end of a slot, marked as $\left(n^{-}, n\right)(n=$ $2,3, \ldots)$.

We assume that the arrival intervals of $\mathrm{PU}$ and SU packets follow geometric distributions with arrival rates $\lambda_{\mathrm{pu}}(0<$ $\left.\lambda_{\mathrm{pu}}<1\right)$ and $\lambda_{\mathrm{su}}\left(0<\lambda_{\mathrm{su}}<1\right)$, respectively. We also define $\bar{\lambda}_{\mathrm{pu}}=1-\lambda_{\mathrm{pu}}$ and $\bar{\lambda}_{\mathrm{su}}=1-\lambda_{\mathrm{su}}$. In this paper, we assume that the transmission time of a PU packet follows geometric distribution with service rate $\mu_{\mathrm{pu}}\left(0<\mu_{\mathrm{pu}}<1\right)$. The PU packet is always being transmitted in a high rate $\mu_{\mathrm{pu}}$, which is not affected by the dual rate transmission strategy.

During an awake period, SU packet is transmitted at a high speed and the transmission time follows geometric distribution with parameter $\mu_{\mathrm{su}}^{u}\left(0<\mu_{\mathrm{su}}^{u}<1\right)$. That is, in a time slot, the transmission of an $\mathrm{SU}$ packet is completed at a high speed with probability $\mu_{\mathrm{su}}^{u}$ and continues to be transmitted with probability $\bar{\mu}_{\mathrm{su}}^{u}=1-\mu_{\mathrm{su}}^{u}$. When the BS is in the working sleep period, the SU packet is transmitted at a low speed, and the transmission time follows geometric distribution with parameter $\mu_{\mathrm{su}}^{v}\left(0<\mu_{\mathrm{su}}^{v}<1\right)$. In addition, we assume that the sleep timer length follows a geometric distribution with parameter $\theta(0<\theta<1)$. Here, we call the parameter $\theta$ as the sleeping parameter.

Assume that the PU packet is not buffered, and the SU packet has a cache with infinite capacity. Let $X_{n}=i(i=$ $0,1, \ldots)$ and $Y_{n}=j(j=0,1,2)$ be the number of secondary packets in the system and in the BS stage, respectively, at the instant $n^{+} . j=0$ means that the BS is in a working sleep period. $j=1$ means that the $\mathrm{BS}$ is awake for the transmission of a PU packet, and $j=2$ means that the BS is awake for the transmission of an $\mathrm{SU}$ packet. We also call the number of SU packets in the system the system level. $\left\{\left(X_{n}, Y_{n}\right), n \geq 1\right\}$ constitutes a two-dimensional discrete time Markov chain (DTMC). The state-space of the DTMC is given as follows:

$$
\boldsymbol{\Omega}=\{(i, j): i \geq 0, j=0,1,2\} .
$$

3.2. Model Analysis. Let $\mathbf{P}$ be the state transition probability matrix of the DTMC $\left\{\left(X_{n}, Y_{n}\right), n \geq 1\right\}$. Let $\mathbf{B}_{r q}$ be the transition probability submatrix for the number of $S U$ packets in the system changing from the system level $r$ to the system level $q$.

When $0 \leq r \leq 1$, the transition probability submatrix of the system level is given as follows.

For the case of $r=0$, if no SU packets arrive at the system at the time, the system level keeps unchanged. The submatrix $\mathbf{B}_{00}$ is given as follows:

$$
\mathbf{B}_{00}=\bar{\lambda}_{\mathrm{su}}\left(\begin{array}{ccc}
\bar{\lambda}_{\mathrm{pu}} & \lambda_{\mathrm{pu}} & 0 \\
\mu_{\mathrm{pu}} \bar{\lambda}_{\mathrm{pu}} & \bar{\mu}_{\mathrm{pu}}+\mu_{\mathrm{pu}} \lambda_{\mathrm{pu}} & 0 \\
0 & 0 & 0
\end{array}\right) .
$$


If an SU packet arrives at the system at the time, the system level increases by one. The submatrix $\mathbf{B}_{01}$ is given as follows:

$$
\mathbf{B}_{01}=\lambda_{\mathrm{su}}\left(\begin{array}{ccc}
\bar{\lambda}_{\mathrm{pu}} \bar{\theta} & \lambda_{\mathrm{pu}} & \bar{\lambda}_{\mathrm{pu}} \theta \\
0 & \bar{\mu}_{\mathrm{pu}}+\mu_{\mathrm{pu}} \lambda_{\mathrm{pu}} & \mu_{\mathrm{pu}} \bar{\lambda}_{\mathrm{pu}} \\
0 & 0 & 0
\end{array}\right)
$$

For the case of $r=1$, if no SU packet arrives at the system and the SU packet in the system departs the system, the system level decreases by one. The submatrix $\mathbf{B}_{10}$ is given as follows:

$$
\mathbf{B}_{10}=\left(\begin{array}{ccc}
\mu_{\mathrm{su}}^{v} \bar{\lambda}_{\mathrm{su}} & 0 & 0 \\
0 & 0 & 0 \\
\mu_{\mathrm{su}}^{u} \bar{\lambda}_{\mathrm{su}} & 0 & 0
\end{array}\right) \times\left(\begin{array}{ccc}
\bar{\lambda}_{\mathrm{pu}} & \lambda_{\mathrm{pu}} & 0 \\
0 & 0 & 0 \\
0 & 0 & 0
\end{array}\right)
$$

If no SU packet arrives at the system and the SU packet in the system does not depart the system, or, an SU packet arrives at the system and the SU packet in the system departs the system, the system level is fixed at 1 .

The submatrix $\mathbf{B}_{11}$ is given as follows:

$$
\begin{aligned}
\mathbf{B}_{11}= & \left(\begin{array}{ccc}
\bar{\mu}_{\mathrm{su}}^{v} \bar{\lambda}_{\mathrm{su}}+\mu_{\mathrm{su}}^{v} \lambda_{\mathrm{su}} & 0 & 0 \\
0 & \bar{\lambda}_{\mathrm{su}} & 0 \\
0 & 0 & \mu_{\mathrm{su}}^{u} \lambda_{\mathrm{su}}+\bar{\mu}_{\mathrm{su}}^{u} \bar{\lambda}_{\mathrm{su}}
\end{array}\right) \\
& \times\left(\begin{array}{ccc}
\bar{\lambda}_{\mathrm{pu}} \bar{\theta} & \lambda_{\mathrm{pu}} & \bar{\lambda}_{\mathrm{pu}} \theta \\
0 & \bar{\mu}_{\mathrm{pu}}+\mu_{\mathrm{pu}} \lambda_{\mathrm{pu}} & \mu_{\mathrm{pu}} \bar{\lambda}_{\mathrm{pu}} \\
0 & \lambda_{\mathrm{pu}} & \bar{\lambda}_{\mathrm{pu}}
\end{array}\right)
\end{aligned}
$$

When the initial level $r>1$, the adjacent levels are $r-1, r$, and $r+1$; the corresponding submatrix is expressed as $\mathbf{B}_{r, r-1}$, $\mathbf{B}_{r r}$, and $\mathbf{B}_{r, r+1}$, respectively. $\mathbf{B}_{r, r-1}, \mathbf{B}_{r r}$, and $\mathbf{B}_{r, r+1}$ are given as follows:

$$
\begin{aligned}
\mathbf{B}_{r, r-1}= & \left(\begin{array}{ccc}
\mu_{\mathrm{su}}^{v} \bar{\lambda}_{\mathrm{su}} & 0 & 0 \\
0 & 0 & 0 \\
\mu_{\mathrm{su}}^{u} \bar{\lambda}_{\mathrm{su}} & 0 & 0
\end{array}\right) \times\left(\begin{array}{ccc}
0 & \lambda_{\mathrm{pu}} & \bar{\lambda}_{\mathrm{pu}} \\
0 & 0 & 0 \\
0 & 0 & 0
\end{array}\right), \\
\mathbf{B}_{r, r}= & \left(\begin{array}{ccc}
\bar{\mu}_{\mathrm{su}}^{v} \bar{\lambda}_{\mathrm{su}} & 0 & \mu_{\mathrm{su}}^{v} \lambda_{\mathrm{su}} \\
0 & \bar{\lambda}_{\mathrm{su}} & 0 \\
0 & 0 & \mu_{\mathrm{su}}^{u} \lambda_{\mathrm{su}}+\bar{\mu}_{\mathrm{su}}^{u} \bar{\lambda}_{\mathrm{su}}
\end{array}\right) \\
& \times\left(\begin{array}{cccc}
\bar{\lambda}_{\mathrm{pu}} \bar{\theta} & \lambda_{\mathrm{pu}} & \bar{\lambda}_{\mathrm{pu}} \theta \\
0 & \bar{\mu}_{\mathrm{pu}}+\mu_{\mathrm{pu}} \lambda_{\mathrm{pu}} & \mu_{\mathrm{pu}} \bar{\lambda}_{\mathrm{pu}} \\
0 & \lambda_{\mathrm{pu}} & \bar{\lambda}_{\mathrm{pu}}
\end{array}\right),
\end{aligned}
$$

$$
\begin{aligned}
\mathbf{B}_{r, r+1}= & \left(\begin{array}{ccc}
\bar{\mu}_{\mathrm{su}}^{v} \lambda_{\mathrm{su}} & 0 & 0 \\
0 & \lambda_{\mathrm{su}} & 0 \\
0 & 0 & \bar{\mu}_{\mathrm{su}}^{u} \lambda_{\mathrm{su}}
\end{array}\right) \\
& \times\left(\begin{array}{ccc}
\bar{\lambda}_{\mathrm{pu}} \bar{\theta} & \lambda_{\mathrm{pu}} & \bar{\lambda}_{\mathrm{pu}} \theta \\
0 & \bar{\mu}_{\mathrm{pu}}+\mu_{\mathrm{pu}} \lambda_{\mathrm{pu}} & \mu_{\mathrm{pu}} \bar{\lambda}_{\mathrm{pu}} \\
0 & \lambda_{\mathrm{pu}} & \bar{\lambda}_{\mathrm{pu}}
\end{array}\right) .
\end{aligned}
$$

Starting from system level 2, all the state transition probability submatrices are repeated forever. For convenience of description, we denote $\mathbf{B}_{r, r-1}=\mathbf{A}_{0}, \mathbf{B}_{r, r}=\mathbf{A}_{1}$, and $\mathbf{B}_{r, r+1}=$ $\mathrm{A}_{2}$.

According to the changes of the system level, the block tridiagonal matrix of one-step transition probability matrix $\mathbf{P}$ is given as follows:

$$
\mathbf{P}=\left(\begin{array}{cccccc}
\mathbf{B}_{00} & \mathbf{B}_{01} & & & & \\
\mathbf{B}_{10} & \mathbf{B}_{11} & \mathbf{A}_{2} & & & \\
& \mathbf{A}_{0} & \mathbf{A}_{1} & \mathbf{A}_{2} & & \\
& & \mathbf{A}_{0} & \mathbf{A}_{1} & \mathbf{A}_{2} & \\
& & & \ddots & \ddots & \ddots
\end{array}\right) .
$$

Let $\pi_{i, j}$ be the steady-state distribution of the twodimensional DTMC. The distribution $\pi_{i, j}$ is then defined as follows:

$$
\pi_{i, j}=\lim _{n \rightarrow \infty} P\left\{X_{n}=i, Y_{n}=j\right\} .
$$

By $\pi_{i}(i \geq 0)$ we denote the vector $\left(\pi_{i, 0}, \pi_{i, 1}, \pi_{i, 2}\right)$; that is, $\pi_{i}=\left(\pi_{i, 0}, \pi_{i, 1}, \pi_{i, 2}\right)$, where $i=0,1,2, \ldots$.

Let $\Pi$ be the steady-state distribution of the system. $\Pi$ is given as follows:

$$
\Pi=\left(\boldsymbol{\pi}_{0}, \boldsymbol{\pi}_{1}, \boldsymbol{\pi}_{2}, \ldots\right) .
$$

Combining the balance equation and the normalization condition for the DTMC, we have

$$
\begin{gathered}
\Pi \mathbf{P}=\Pi, \\
\Pi \mathbf{e}=1,
\end{gathered}
$$

where $\mathbf{e}$ is a column vector with ones.

From the state transition probability matrix $\mathbf{P}$, we find that the system transitions occur only between adjacent levels. The Markov chain $\left\{\left(X_{n}, Y_{n}\right), n \geq 1\right\}$ can be seen as a quasi-birth-death process. Therefore, matrix-geometric solution [17] method can be used to obtain the steady-state probability distribution of system.

Let $\mathbf{R}$ be the minimal nonnegative solution of the matrix quadratic equation $\mathbf{R}^{2} \mathbf{A}_{0}+\mathbf{R} \mathbf{A}_{1}+\mathbf{A}_{2}=\mathbf{R}$, and the spectral radius $\mathrm{sp}(\mathbf{R})<1$. We construct a stochastic matrix $B[\mathbf{R}]$ with positive left invariant vector as follows:

$$
B[\mathbf{R}]=\left(\begin{array}{cc}
\mathbf{B}_{00} & \mathbf{B}_{01} \\
\mathbf{B}_{10} & \mathbf{B}_{11}+\mathbf{R} \mathbf{A}_{0}
\end{array}\right) .
$$


Then, $\boldsymbol{\pi}_{0}$ and $\boldsymbol{\pi}_{1}$ satisfy the following equation:

$$
\begin{aligned}
\left(\boldsymbol{\pi}_{0}, \boldsymbol{\pi}_{1}\right) & =\left(\boldsymbol{\pi}_{0}, \boldsymbol{\pi}_{1}\right) B[\mathbf{R}], \\
\boldsymbol{\pi}_{0} \mathbf{e}+\boldsymbol{\pi}_{1}(\mathbf{I}-\mathbf{R})^{-1} \mathbf{e} & =1,
\end{aligned}
$$

where $\mathbf{e}$ is a column vector with ones.

By using the Gauss-Seidel method [18] to solve (12), we can obtain $\boldsymbol{\pi}_{0}$ and $\boldsymbol{\pi}_{1}$. Combining with $\boldsymbol{\pi}_{k}=\boldsymbol{\pi}_{1} \mathbf{R}^{k-1}, k \geq 2$, the steady-state distribution of the system $\Pi$ can be presented numerically.

\section{System Performance Measures}

Based on the steady-state distribution of the system model, the performance measures of the dual rate energy saving strategy are derived.

(1) We define the latency time of a SU packet as the duration from the instant the SU packet enters the system to the instant the SU packet is transmitted successfully. From the total probability formula, the average queue length in steadystate is found to be equal to $\sum_{i=1}^{\infty} \sum_{j=0}^{2} i \pi_{i, j}$. Using Little's law, the average latency time $W_{\text {su }}$ of $S U$ packets is given as follows:

$$
W_{\mathrm{su}}=\frac{1}{\lambda_{\mathrm{su}}} \sum_{i=1}^{\infty} \sum_{j=0}^{2} i \pi_{i, j} .
$$

(2) The forced termination probability $\gamma$ of SU packets is defined as the number of SU packets with interrupt transmission per unit time slot due to the arrival of the PU packet. The probability that the SU packet will be transmitted at a low rate is $\sum_{i=1}^{\infty} \pi_{i, 0} \bar{\mu}_{\mathrm{su}}^{v}$, and at a high rate it is $\sum_{i=1}^{\infty} \pi_{i, 2} \bar{\mu}_{\mathrm{su}}^{u}$. The forced termination probability $\gamma$ of SU packet is given as follows:

$$
\gamma=\lambda_{\mathrm{pu}}\left(\sum_{i=1}^{\infty} \pi_{i, 0} \bar{\mu}_{\mathrm{su}}^{v}+\sum_{i=1}^{\infty} \pi_{i, 2} \bar{\mu}_{\mathrm{su}}^{u}\right) .
$$

(3) The channel utilization rate $\phi$ is defined as the probability that the channel be occupied by user data packets (PU packet or SU packet) within a slot. The probability that the SU packet occupies the channel is $\sum_{i=1}^{\infty} \pi_{i, 0}+\sum_{i=1}^{\infty} \pi_{i, 2}$; the probability that the PU packet occupies the channel is $\sum_{i=0}^{\infty} \pi_{i, 1}$. The channel utilization rate is then given as follows:

$$
\phi=\sum_{i=1}^{\infty} \pi_{i, 0}+\sum_{i=1}^{\infty} \pi_{i, 2}+\sum_{i=0}^{\infty} \pi_{i, 1}=1-\pi_{0,0} .
$$

(4) The energy saving ratio $\delta$ is defined as the energy conservations per slot due to the introduction of dual rate transmission strategy. The energy consumption of the BS in the high speed transmission state is the same as that of the BS without dual rate transmission strategy. Let $C_{H}$ be the energy consumption per slot when the BS is in a high speed transmission state. Assuming that the BS energy consumption is proportional to the transmission rate, the energy consumption per slot is $C_{L}=\left(\mu_{\mathrm{su}}^{v} / \mu_{\mathrm{su}}^{u}\right) C_{H}$ when the system is in a low speed transmission. In the working sleep
TABLE 1: System parameters.

\begin{tabular}{lc}
\hline Parameter names & Parameter values \\
\hline $\begin{array}{l}\text { Physical layer transfer rate } \\
\text { Slot }\end{array}$ & $20 \mathrm{Mbps}$ \\
Mean value of PU packet size & $1 \mathrm{~ms}$ \\
Mean value of SU packet size & 3745 bytes \\
$\begin{array}{l}\text { Energy consumption (EC) per slot in high } \\
\text { speed transmission }\end{array}$ & 4277 bytes \\
$\begin{array}{l}\text { EC per slot in the absence of data transmission } \\
\text { EC during listening process at the beginning of } \\
\text { slot when there are no packets to transmit }\end{array}$ & $0.8 \mathrm{~mJ}$ \\
\hline
\end{tabular}

period and without data transmission period, the BS is in a state of fully sleep, and the energy consumption per slot is $C_{0}$. Furthermore, in a fully sleep state, the energy consumption of listening process of the $\mathrm{BS}$ at the beginning of each slot is $C_{L}$. The energy saving ratio $\delta$ with dual rate transmission strategy is given as follows:

$$
\begin{aligned}
\delta & =\left(C_{H}-C_{m}-C_{0}\right) \pi_{0,0}+\left(C_{H}-C_{L}\right) \sum_{i=1}^{\infty} \pi_{i, 0} \\
& =\left(C_{H}-C_{m}-C_{0}\right) \pi_{0,0}+\left(1-\frac{\mu_{\mathrm{su}}^{v}}{\mu_{\mathrm{su}}^{u}}\right) C_{H} \sum_{i=1}^{\infty} \pi_{i, 0} .
\end{aligned}
$$

\section{Numerical Experiments and Discussion}

The analysis results are obtained based on (11) using Matlab 2011a. The simulation results are obtained by averaging over 10 independent runs using MyEclipse 2014. In the numerical experiments, the parameters are set in Table 1 . Based on system parameters, such as arrival rate of SU packets, the low transmission rate of $\mathrm{SU}$ packets, the high transmission rate of SU packets, the arrival rate of PU packets, the transmission rate of PU packets, and the sleeping parameter, we initialize the arrival instants of packets, the service time of each packet, and the time length of the working sleep period. Applying the event-driven methodology, we estimate the latency of each packet and the time duration of each working sleep period.

According to the wireless transmission standard [4] in IEEE 802.22, the experimental parameters are set as shown in Table 1.

By setting different arrival rate $\lambda_{\mathrm{su}}$ of SU packets, arrival rate $\lambda_{\mathrm{pu}}$ of PU packets, and low transmission rate $\mu_{\mathrm{su}}^{v}$, we examine the influence of the sleeping parameter $\theta$ on the average latency of the SU packets $W_{\text {su }}$ in Figure 3.

For the given arrival rate $\lambda_{\text {su }}$ of SU packets, arrival rate $\lambda_{\mathrm{pu}}$ of PU packets, and low transmission rate $\mu_{\mathrm{su}}^{v}$, the average latency of SU packets shows a downward trend with the increase in the working sleep parameter $\theta$. This is because the larger the working sleep parameter is, the smaller the average length of the working sleep timer is, and the shorter the time of the BS in the low speed transmission period is; then the average packet delay of SU packets will decrease.

For the same working sleep parameter $\theta$, the influences of other three parameters on the average latency of $\mathrm{SU}$ 


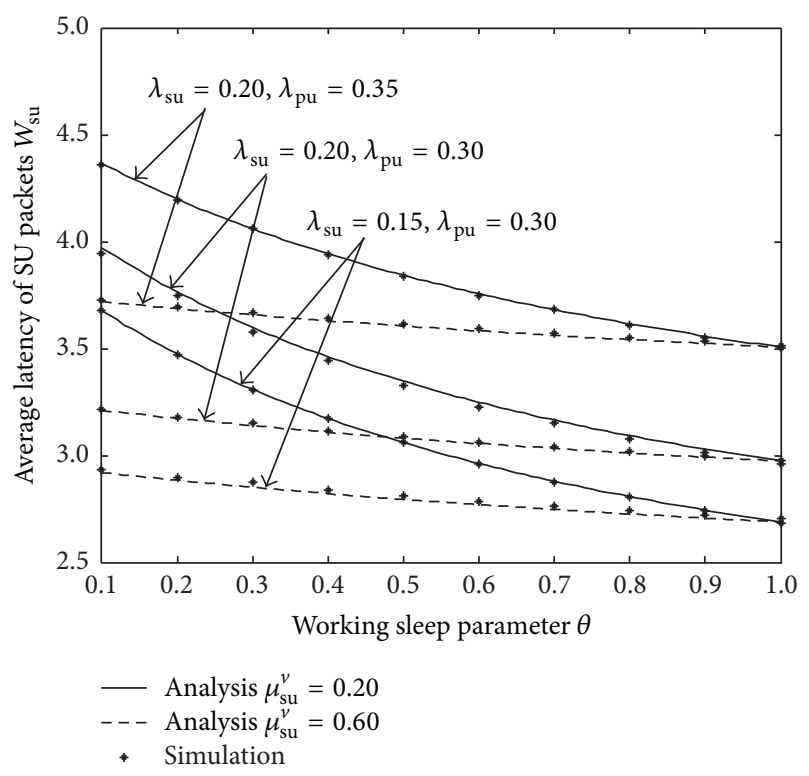

FIGURE 3: The change trend of average latency of SU packets.

packets are addressed as follows: (I) with the increase in the arrival rate $\lambda_{\text {su }}$ of SU packets, the number of SU packets in the system increases, which makes the average waiting time of SU packets longer, and the average latency of SU packets increases accordingly. (II) The number of PU packets increases with the arrival rate $\lambda_{\mathrm{pu}}$ of the PU packets. The more the PU packets in the system are, the longer the SU packets wait for the available channel, and the average packet delay of SU packet increases accordingly. (III) The average transmission time of the SU packet is shortened as the low transmission rate $\mu_{\mathrm{su}}^{v}$ increases, so that the average latency of the SU packet is reduced.

Figure 4 shows the changing trend of the forced termination probability $\gamma$ of SU packets for different arrival rates $\lambda_{\text {su }}$ of $S U$ packets, the arrival rates $\lambda_{\mathrm{pu}}$ of PU packets, and the low transfer rate $\mu_{\mathrm{su}}^{v}$.

With the same parameters of the arrival rate $\lambda_{\text {su }}$ of SU packets, the arrival rate $\lambda_{\mathrm{pu}}$ of PU packets, and the low transmission rate of $\mu_{\mathrm{su}}^{v}$, the forced termination probability $\gamma$ of SU packets shows a downward trend with the increase in the sleep parameter $\theta$. The larger the working sleep parameter is, the smaller the average length of the working sleep timer is, the longer the BS is in the high speed transmission period, and the shorter the time it takes for the SU packet to complete the transmission. As a result, what is less likely is that the arrival of PU packets occurs during the SU packet transmission procedure, and the forced termination probability shows a downward trend.

For the same working sleep parameter $\theta$, the influences of the other three parameters on the forced interruption probability are addressed as follows: (I) with the increase in arrival rate $\lambda_{\text {su }}$ of SU packets, the probability of SU packets occupying channels increases, while the forced termination probability of SU packets increases. (II) The greater the arrival rate $\lambda_{\mathrm{pu}}$ of the packets is, the greater the possibility of the

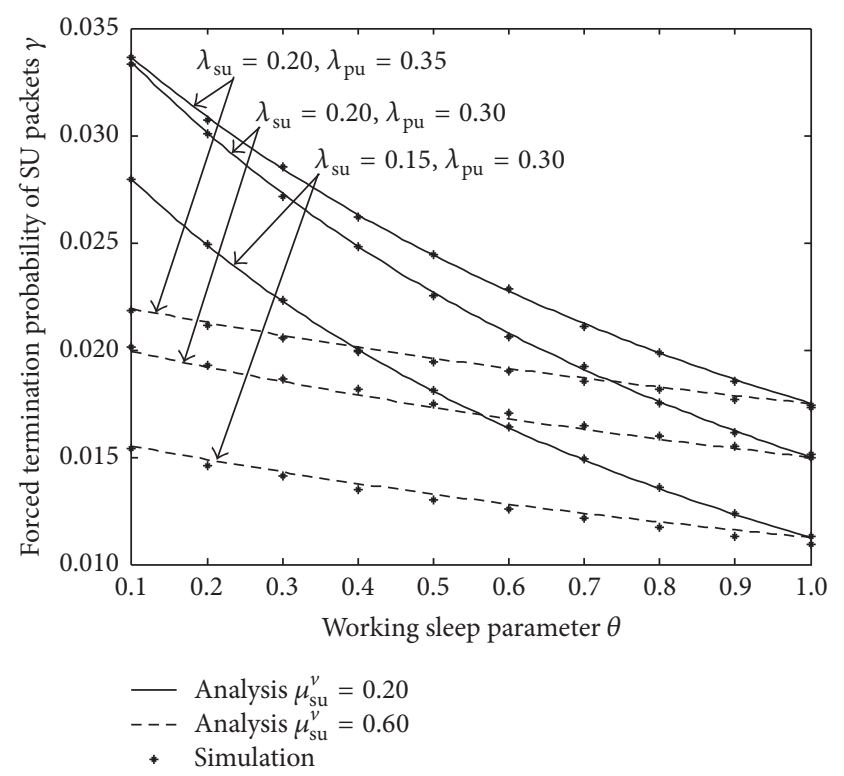

FIGURE 4: The change trend of forced termination probability of SU packets.

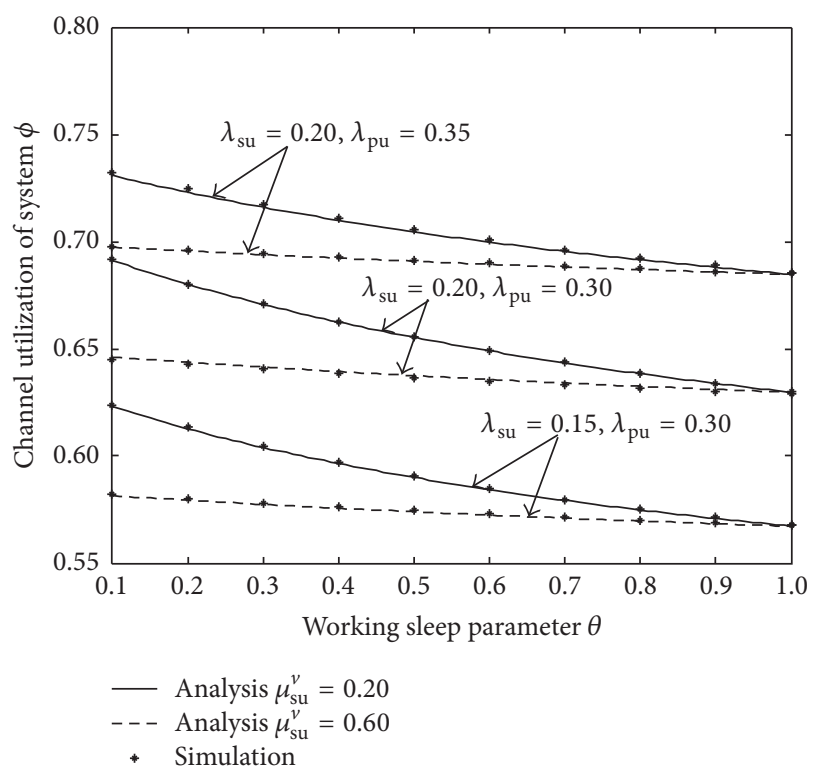

FIGURE 5: The change trend of channel utilization of system.

arrival of the PU data packet during the transmission period of the SU packets is; the forced termination probability of the SU packets will increase. (III) The transmission time of an SU packet decreases with increases in the low transmission rate $\mu_{\mathrm{su}}^{v}$; what is more possible is that the channel is idle when a PU packet arrives; the forced termination probability of SU packets will be greater.

Figure 5 shows the variation trend of the channel utilization rate $\phi$ with the sleep parameter $\theta$ for different arrival rate $\lambda_{\text {su }}$ of SU packets, the arrival rate $\lambda_{\text {pu }}$ of PU packets, and the low transmission rate $\mu_{\mathrm{su}}^{v}$. 


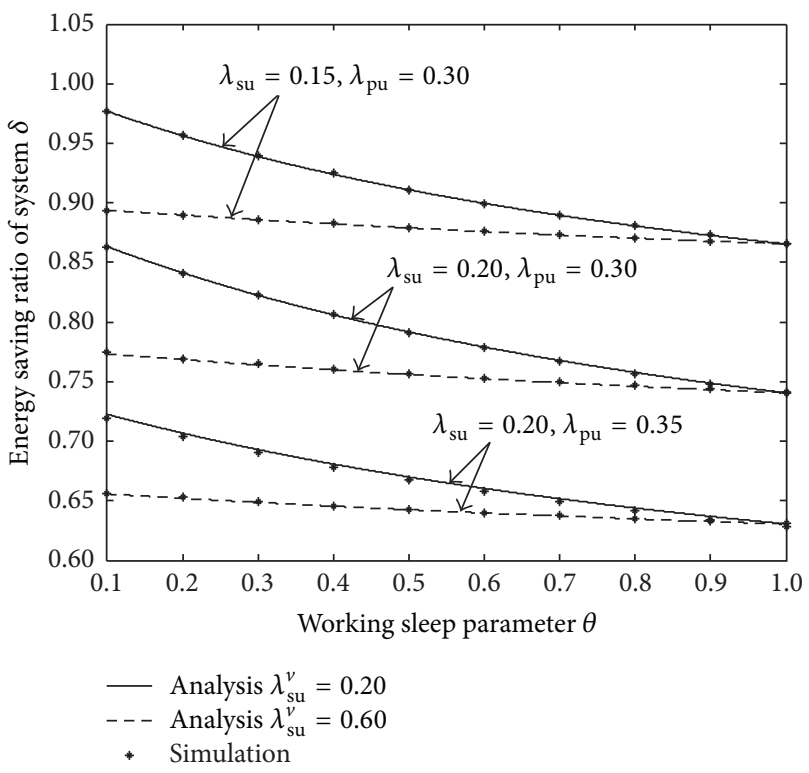

FIGURE 6: The change trend of energy saving ratio of system.

For a given arrival rate $\lambda_{\text {su }}$ of SU packets, arrival rate $\lambda_{\text {pu }}$ of PU packets, and low transmission rate $\mu_{\mathrm{su}}^{v}$, the channel utilization rate $\phi$ decreases with the increase in the working sleep parameter $\theta$. The bigger the working sleep parameter is, the smaller the average length of working sleep timer is, and the longer the BS is at the high speed transmission state, and an SU packet transmission requires shorter time, so the channel utilization decreases.

For the same working sleep parameter $\theta$, the influences of other three parameters on the channel utilization are addressed as follows: (I) with the increase in arrival rate $\lambda_{\text {su }}$ of SU packets, what is more possible is that the channel is occupied by SU packets. (II) The number of PU packets in the system increases with the increase of the arrival rate $\lambda_{\mathrm{pu}}$ of the PU packets, so that the channel is more likely to be occupied. (III) The transmission time of an SU packet increases as the low transmission rate $\mu_{\mathrm{su}}^{v}$ decreases, and the SU packets will occupy the channel for a longer time.

Figure 6 displays the changing trends of the energy saving ratio $\delta$ of system for different arrival rate $\lambda_{\text {su }}$ of SU packets, arrival rate $\lambda_{\mathrm{pu}}$ of PU packets, and the low transmission rate $\mu_{\mathrm{su}}^{v}$.

With the same system parameters in terms of the arrival rate $\lambda_{\text {su }}$ of SU packets, the arrival rate $\lambda_{\text {pu }}$ of the PU packets, and the low transmission rate $\mu_{\mathrm{su}}^{v}$, the energy saving ratio $\delta$ decreases with the increase in the working sleep parameter $\theta$. This is because the larger the working sleep parameter is, the smaller the average length of the working sleep timer is, and the shorter the time the BS is in the energy saving state, so the energy savings ratio is on the downward trend.

For the same working sleep parameter $\theta$, the influences of the other three parameters on the energy saving ratio are addressed as follows: (I) with the increase in the arrival rate $\lambda_{\text {su }}$ of SU packets, the number of SU packets in the system increases, and the system stays a shorter time during energy saving state, so the energy saving rate is decreased. (II) The number of PU packets in the system increases with the arrival rate $\lambda_{\text {pu }}$ of the PU packets, while the PU packets are more likely transmitted in the high speed transmission period, so the energy saving rate is reduced. (III) The greater the low transmission rate $\mu_{\mathrm{su}}^{v}$ is, the higher the energy consumption in the low speed transmission period is, so the energy saving ratio is decreased.

Summarizing the experimental results in Figures 3-6, we draw a conclusion that when the working sleep parameter and the low transmission rate increase, the average latency and the forced termination probability of the SU packets decrease, but the channel utilization and energy saving ratio also show a downward trend. In fact, all the SU packets have their respective profit and are independent of whether or not they occupy free channels; in other words, they are selfish. Each packet's objectives can be considered as maximizing its respective net profit.

\section{Performance Optimization and Pricing Policy}

A strategy profile is a Nash equilibrium if no data packets can do better by unilaterally changing its strategy. Because PU packets are authorized to occupy the spectrum by charging, the transmission of the PU packets is not affected by SU packets, according to their own needs for transmission. However, SU packets occupy the spectrum in opportunistic approach. Every SU packet occupies the spectrum as much as possible to transmit data, so there is a game behavior between the SU packets. For the above reasons, we investigated the Nash equilibrium for SU packets but not for PU packets.

In this paper, the system performance is optimized from the point of view of arrival rate of SU packets. In order to analyze the Nash equilibrium and the social optimal behavior of the SU packets under dual rate energy saving strategy, the following assumptions are given as follows:

(1) SU packets cannot obtain any information (number of SU packets in the cache, status of the current channel, etc.) of the system before they enter the cache.

(2) When an SU packet is successfully transmitted, the reward is $G$, and the cost for waiting in the system is $C$ per unit slot.

(3) The individual net profit function of the SU packets is identical and can be added together.

(4) All the SU packets obey the rule of first come first served (FCFS).

\subsection{Performance Optimization}

6.1.1. Nash Equilibrium Behavior. Once an SU packet enters the cache, it can leave the system only when it is successfully completed. SU packets are risk neutral; that is, they will maximize the expected net profit. Then, the individual benefit function of an SU packet is given as follows:

$$
U_{i}=G-C W_{\mathrm{su}}
$$

where $W_{\text {su }}$ is the average latency of SU packets. 
In order to ensure that the system is in steady-state, we set the upper bound of the arrival rate of SU packets as $\lambda_{\mathrm{su}}^{\max }$ and the lower bound as $\lambda_{\mathrm{su}}^{\mathrm{min}}\left(\lambda_{\mathrm{su}}^{\mathrm{min}} \geq 0\right)$. Within constraint range $\left[\lambda_{\mathrm{su}}^{\mathrm{min}}, \lambda_{\mathrm{su}}^{\mathrm{max}}\right]$, as the arrival rate of SU packets increases, the average delay of SU packets increases, and the net profit of an SU packet decreases. Since SU packets are risk neutral, they will try their best to access the channel. Under the condition of $\lambda_{\mathrm{su}}^{\mathrm{min}} \geq 0$, the arrival rate of SU packets is as large as possible and eventually reaches the Nash equilibrium state. Any SU packet has no motive to break the current equilibrium state, when the individually optimal arrival rate of the SU packet is $\lambda_{\mathrm{su}}^{e}$.

Under the dual rate energy saving strategy, the Nash equilibrium behavior of the SU packets is as follows:

(1) $\lambda_{\mathrm{su}}=\lambda_{\mathrm{su}}^{\mathrm{min}}$ and $U_{i} \leq 0$. In this case, even if no new SU packet enters the cache, the net profit of the SU packets in the system is also negative. At this point, the individually optimal arrival rate of the SU packet is $\lambda_{\mathrm{su}}^{e}=\lambda_{\mathrm{su}}^{\mathrm{min}}$.

(2) $\lambda_{\mathrm{su}}=\lambda_{\mathrm{su}}^{\mathrm{max}}$ and $U_{i} \geq 0$. In this case, even if all SU packets enter the cache, the net profit of each SU packet is positive. At this point, the individually optimal arrival rate of the SU packet is $\lambda_{\mathrm{su}}^{e}=\lambda_{\mathrm{su}}^{\max }$.

(3) $\lambda_{\mathrm{su}}=\lambda_{\mathrm{su}}^{\mathrm{min}}$ and $U_{i} \geq 0$ and $\lambda_{\mathrm{su}}=\lambda_{\mathrm{su}}^{\mathrm{max}}$ and $U_{i} \leq$ 0 . In this case, only partial SU packets enter the cache to guarantee $U_{i} \geq 0$. When the net profit is zero, the system reaches the Nash equilibrium; that is, $U_{i}=0$. At this point, the individually optimal arrival rate of the SU packet is $\lambda_{\mathrm{su}}^{\mathrm{min}}<$ $\lambda_{\mathrm{su}}^{e}<\lambda_{\mathrm{su}}^{\max }$.

6.1.2. Socially Optimal Behavior. From the social point of view, the performance of the system is analyzed and its social optimization is carried out. On the premise that the cost of the SU packets in system is not charged, the sum of the personal profit of all SU packets in a slot is the social profit; we have

$$
U_{\text {so }}=\lambda_{\text {su }} U_{i}=\lambda_{\text {su }}\left(G-C W_{\text {su }}\right) .
$$

By maximizing the social profit $U_{\text {so }}$ in formula (18), we can get the optimal social rate of $\lambda_{\mathrm{su}}^{*}$ as follows:

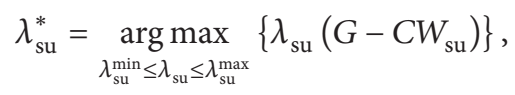

where arg max means finding the arrival rate $\lambda_{\text {su }}^{*}$ of the SU packet that makes $U_{\text {so }}$ maximum.

Using the experimental parameters in Table 1 and setting $G=5, C=1$ as an example, we show social profit changes with the arrival rate of SU packets in Figure 7.

In Figure 7, we find that when the arrival rate of SU packets is smaller, with the increase in the arrival rate of SU packets, the social profit shows an upward trend. When the arrival rate of SU packets is larger, with the increase in the number of SU packets, the social profit shows a downward trend. Therefore, there is the social optimal arrival rate and the maximum social profit.

From (18), we see that $U_{i}=0$ when $U_{\text {so }}=0$. The individual optimal arrival rate $\lambda_{\mathrm{su}}^{e}$ corresponding to $U_{\text {so }}=0$ is marked with " $\square$ "; the social optimal rate $\lambda_{\text {su }}^{*}$ corresponding to the maximum value of $U_{\text {so }}$ is marked with " $\mathbf{}$."

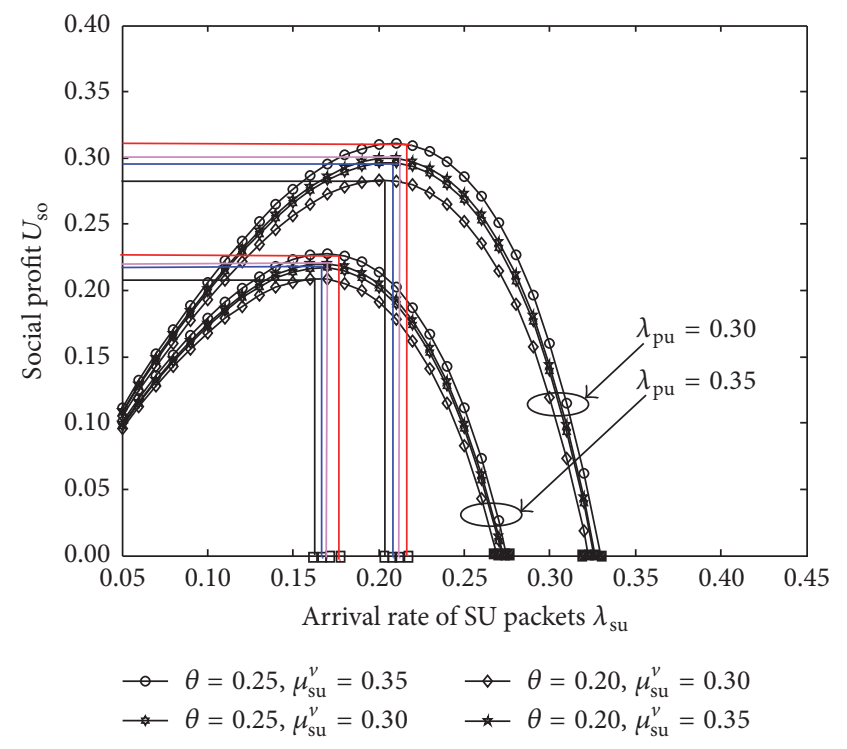

FIGURE 7: The change trend of social profit.

By observing the trend of social profit shown in Figure 7, we can get $\lambda_{\mathrm{su}}^{*} \leq \lambda_{\mathrm{su}}^{e}$. This means that in the case of just considering individual net profit, more SU packets will arrive at the system. In order to make the individual optimal arrival rate equal to the social optimal arrival rate, it is necessary to formulate a proper charging scheme with admission fee for the SU packets. The admission fee is important to coincide the Nash equilibrium and the socially optimization.

6.2. Pricing Policy. In order to formulate a reasonable pricing policy, we need to find the socially optimal arrival rate first.

6.2.1. Optimization Algorithm. It can be seen from Figure 7 that the social profit function is continuously nonlinear and it is difficult to obtain the social optimal arrival rate. Therefore, an intelligent optimization algorithm based on particle swarm optimization [19] is introduced. The process of particle movement is the searching process of the optimal packet arrival rate. After several moves, the particles finally converge to the optimal position, that is, the optimal arrival rate. During the optimization process, the speed and magnitude of each arrival rate are adjusted repeatedly to make the arrival rate oscillate randomly around the local optimal and the global optimal position. Based on the above idea, we present an algorithm as follows.

Algorithm 1 (algorithm to obtain optimal arrival rate).

Input. The number $N$ of the arrival rates of SU packets, upper bound $\lambda_{\mathrm{su}}^{\mathrm{max}}$ and lower bound $\lambda_{\mathrm{su}}^{\mathrm{min}}$ for the arrival rates of SU packets, acceleration constant $c_{1}, c_{2} \in(0,2)$, the minimum $\omega_{\min }$ and the maximum $\omega_{\max }$ of inertia weight, the maximum number iter ${ }_{\max }$ of iterations, and the maximum speed $V_{\mathrm{su}}^{\max }$ are the input.

Output. The socially optimal arrival rate $\lambda_{\text {su }}^{*}$ is the output. 
TABLE 2: Numerical results of the pricing policy.

\begin{tabular}{|c|c|c|c|c|}
\hline $\begin{array}{l}\text { Arrival rate of PU package } \\
\lambda_{\mathrm{pu}}\end{array}$ & $\begin{array}{l}\text { Working sleep parameter } \\
\theta\end{array}$ & $\begin{array}{l}\text { Low transmission speed } \\
\mu_{\mathrm{su}}^{v}\end{array}$ & $\begin{array}{c}\text { Socially optimal arrival rate } \\
\qquad \lambda_{\mathrm{su}}^{*}\end{array}$ & $\begin{array}{l}\text { Admission fee } \\
p \\
\end{array}$ \\
\hline \multirow{4}{*}{0.30} & \multirow{2}{*}{0.20} & 0.30 & 0.2024 & 1.4005 \\
\hline & & 0.35 & 0.2058 & 1.4574 \\
\hline & \multirow{2}{*}{0.25} & 0.30 & 0.2053 & 1.4432 \\
\hline & & 0.35 & 0.2083 & 1.4925 \\
\hline \multirow{4}{*}{0.35} & \multirow{2}{*}{0.20} & 0.30 & 0.1642 & 1.2712 \\
\hline & & 0.35 & 0.1670 & 1.3186 \\
\hline & \multirow{2}{*}{0.25} & 0.30 & 0.1665 & 1.3057 \\
\hline & & 0.35 & 0.1690 & 1.3471 \\
\hline
\end{tabular}

Step 1. Generate two random numbers as the initial values of the $i$ th $(i=1,2, \ldots, N)$ arrival rate $\lambda_{\mathrm{su}}^{i} \in\left[\lambda_{\mathrm{su}}^{\min }, \lambda_{\mathrm{su}}^{\max }\right]$ and the speed $v_{\mathrm{su}}^{i} \in[0,1]$ of the SU packets, respectively. Set the initial value of the iteration number as iter $=0$.

Step 2. Calculate the social profit of each arrival rate $\lambda_{\mathrm{su}}^{i}(i=$ $1,2, \ldots, N)$ of SU packets as follows:

$$
U_{\mathrm{so}}^{i}=\lambda_{\mathrm{su}}^{i}\left(G-C W_{\mathrm{su}}^{i}\right),
$$

$\% W_{\text {su }}^{i}$ is the average latency of SU packets with the arrival rate $\lambda_{\mathrm{su}}^{i} \%$

Step 3. Find the maximum social profit $\max _{\text {so }}^{i}$ and the corresponding local optimal arrival rate $p_{\mathrm{su}}^{i}$ of the $i$ th $(i=$ $1,2, \ldots, N)$ arrival rate of SU packets.

Step 4. Determine the maximum social profit in the searching space and record the corresponding global optimal arrival rate $p_{\mathrm{su}}^{g}$.

$$
p_{\mathrm{su}}^{g}=\underset{i=1,2, \ldots, N}{\arg \max }\left\{\max _{\mathrm{so}}^{i}\right\}
$$

Step 5. Calculate inertia weight $\omega$.

$$
\omega=\omega_{\max }-\frac{\omega_{\max }-\omega_{\min }}{\text { iter }_{\max }} \times \text { iter. }
$$

Step 6. Update the $i$ th $(i=1,2, \ldots, N)$ arrival rate of SU packets.

$$
\begin{aligned}
& v_{\mathrm{su}}^{i}=\omega v_{\mathrm{su}}^{i}+c_{1} m_{1}\left(p_{\mathrm{su}}^{i}-\lambda_{\mathrm{su}}^{i}\right)+c_{2} m_{2}\left(p_{\mathrm{su}}^{g}-\lambda_{\mathrm{su}}^{i}\right), \\
& \lambda_{\mathrm{su}}^{i}=\lambda_{\mathrm{su}}^{i}+v_{\mathrm{su}}^{i} .
\end{aligned}
$$

$\% m_{1}=$ Random and $m_{2}=$ Random, where Random is a random number between 0 and 1 , and $v_{\mathrm{su}}^{i} \in\left[-V_{\mathrm{su}}^{\mathrm{max}},+V_{\mathrm{su}}^{\mathrm{max}}\right] . \%$

Step 7. Update iterative variable as iter $=$ iter +1 :

$$
\begin{aligned}
& \text { If iter } \leq \text { iter }_{\max } \\
& \text { then go to Step } 3 . \\
& \text { else } \lambda_{\mathrm{su}}^{*}=p_{\mathrm{su}}^{g}, \\
& \text { endif. }
\end{aligned}
$$

Step 8. Output $\lambda_{\mathrm{su}}^{*}$.
6.2.2. Admission Fee. By introducing a pricing policy for SU packets, we set a spectrum admission fee $p$. The individual benefit function of an SU packet is modified as follows:

$$
U_{i}^{\prime}=G-C W_{\mathrm{su}}-p .
$$

Recalculate social profit as

$$
U_{\text {so }}^{\prime}=\lambda_{\text {su }}\left(G-C W_{\text {su }}-p\right)+\lambda_{\text {su }} p=\lambda_{\text {su }}\left(G-C W_{\text {su }}\right) \text {. }
$$

Comparing (18) and (25), we find that the final expressions for $U_{\text {so }}$ and $U_{\text {so }}^{\prime}$ are the same. This is because that the aggregate of the spectrum admission fee is still in the system, in spite of there being a pricing policy for SU packets. In other words, the fee is just transferred from the SU packets to the BS. As a result, the appropriate pricing policy can be formulated, so that the social optimal arrival rate is consistent with the individual optimal arrival rate.

Substituting the social optimal arrival rate $\lambda_{\text {su }}^{*}$ obtained by the above algorithm into (24), the admission fee of SU packets is given as follows:

$$
p=G-C W_{\mathrm{su}}^{*},
$$

where $W_{\mathrm{su}}^{*}$ is the average latency of SU packets with the socially optimal arrival rate $\lambda_{\mathrm{su}}^{*}$.

For different arrival rate $\lambda_{\mathrm{pu}}$ of PU packets, the working sleep parameter $\theta$, and the low transfer rate $\mu_{\mathrm{su}}^{v}$, the numerical results of the social optimal arrival rate $\lambda_{\mathrm{su}}^{*}$ and the admission fee $p$ of SU packets are shown in Table 2 .

Conclusions can be drawn from the numerical results in Table 2: (I) with the increase in the arrival rate of the PU packets, the average latency of SU packets in the system increases, and so the number of SU packets entering the cache decreases. In order to encourage more SU packets to enter the cache and achieve the maximum social profit, the admission fee should be set lower. (II) With the increase in working sleep parameter, the length of working sleep period is decreased, the average packet delay of SU packets is decreased, and more SU packets will enter the cache. In order to improve the response performance of the system and maximize the social profit, the admission fee can be set higher. (III) The larger the low transmission rate is, the shorter the average delay of SU packets is, and the more the SU packets will enter the cache. Similarly, in order to improve the system performance and maximize the social profit, the admission fee should be set higher. 


\section{Conclusion}

A new dual rate transmission strategy is proposed to reduce the energy consumption of communication networks for wireless regional area networks based on IEEE 802.22 standard. Under the constraint of spectrum resource utilization, energy savings are realized by introducing the sleep mechanism into the BS of the network. By means of quasi-birthdeath process and matrix-geometric solution method, the average latency, and forced termination probability of the SU packet, the energy saving ratio and the channel utilization of the system are given. Experimental results show that the dual rate transmission strategy can effectively reduce the energy consumption and guarantee the response performance of the system.

The profit function based on revenue-expenditure structure is established; then the Nash equilibrium and social optimal behavior under the energy saving strategy are investigated. The particle swarm optimization is applied to the profit function; an intelligent optimization algorithm for searching the optimal social arrival rate of SU packets is proposed. Through the formulation of reasonable pricing policy, the individual and social optimums are coincided.

This paper provides a new idea for green communications in computer networks and provides a theoretical basis for the performance optimization of cognitive radio networks. In the future, we will study the performance of WRAN by considering more factors to measure the energy consumption per unit time.

\section{Conflicts of Interest}

The authors declare that they have no conflicts of interest.

\section{Acknowledgments}

This work was supported by the National Natural Science Foundation of China (Grant nos. 61472342 and 61572379).

\section{References}

[1] The Climate Group, Smart 2020: Enabling the low carbon economy in the information age [EB/OL], 2008, http://www.theclimategroup.org.

[2] A. Dejonghe, B. Bougard, S. Pollin et al., "Green reconfigurable radio systems," IEEE Signal Processing Magazine, vol. 24, no. 3, pp. 90-101, 2007.

[3] K. Hyunduk, H. Heonjin, and K. Changjoo, "Performance evaluation of a CR-based WRAN system using spectrum utilization efficiency," in Proceedings of the 2008 International Conference on Wireless Communications, Networking and Mobile Computing, WiCOM 2008, China, October 2008.

[4] C. Stevenson, G. Chouinard, Z. Lei, W. Hu, S. Shellhammer, and W. Caldwell, "IEEE 802.22: the first cognitive radio wireless regional area network standard," IEEE Communications Magazine, vol. 47, no. 1, pp. 130-138, 2009.

[5] V. Balaji, P. Kabra, P. V. P. K. Saieesh, C. Hota, and G. Raghurama, "Cooperative Spectrum Sensing in Cognitive Radios
Using Perceptron Learning for IEEE 802.22 WRAN," in Proceedings of the 11th International Conference on Communication Networks, ICCN 2015, pp. 14-23, India, August 2015.

[6] A. Bagwari, J. Kanti, G. S. Tomar, and A. Samarah, "Reliable spectrum sensing scheme based on dual detector with doublethreshold for IEEE 802.22 WRAN," Journal of High Speed Networks, vol. 21, no. 3, pp. 205-220, 2015.

[7] G. P. Joshi, S. Acharya, and S. W. Kim, "Fuzzy-logic-based channel selection in IEEE 802.22 WRAN," Information Systems, vol. 48, pp. 327-332, 2015.

[8] J. Premalatha, U. Anitha, V. Manonmani, and P. Ganesan, "Survey on energy saving methods for green communication network," Indian Journal of Science \& Technology, vol. 8, no. 19, pp. 1-5, 2015.

[9] G. Rizzo, B. Rengarajan, and M. Ajmone Marsan, "The value of BS flexibility for QoS-aware sleep modes in cellular access networks," in Proceedings of the 2014 IEEE International Conference on Communications Workshops, ICC 2014, pp. 883-888, Australia, June 2014.

[10] G. Wu, L. Dong, Z. Qin, and Z. Xu, "Dynamic programmingbased pico base station sleep mode control in heterogeneous networks," International Journal of Communication Systems, vol. 30, no. 2, Article ID e2967, 2017.

[11] B. Lannoo, A. Dixit, S. Lambert, D. Colle, and M. Pickavet, "How sleep modes and traffic demands affect the energy efficiency in optical access networks," Photonic Network Communications, vol. 30, no. 1, pp. 85-95, 2015.

[12] F. Wu and Z. Zheng, "Game theory based spectrum dynamic management," Journal of Computer Research and Development, vol. 53, no. 1, pp. 38-52, 2016, (in Chinese).

[13] G. Wang and Z. Zeng, "Price-based spectrum access algorithm in cognitive radio networks," Journal of Harbin University of Science and Technology, vol. 19, no. 2, pp. 115-119, 2014, (in Chinese).

[14] H. Li and Z. Han, "Socially optimal queuing control in cognitive radio networks subject to service interruptions: to queue or not to queue?" IEEE Transactions on Wireless Communications, vol. 10, no. 5, pp. 1656-1666, 2011.

[15] Y. Xie, X. Tan, L. Ma et al., "Spectrum allocation algorithm base on distributed game theory," Systems Engineering and Electronics, vol. 37, no. 10, pp. 2391-2395, 2015, (in Chinese).

[16] N. H. Tran, C. S. Hong, Z. Han, and S. Lee, "Optimal pricing effect on equilibrium behaviors of delay-sensitive users in cognitive radio networks," IEEE Journal on Selected Areas in Communications, vol. 31, no. 11, pp. 2566-2579, 2013.

[17] A. S. Alfa, Queueing Theory for Telecommunications, Springer, New York, USA, 2010, 65-78.

[18] H. Niki, K. Harada, M. Morimoto, and M. Sakakihara, "The survey of preconditioners used for accelerating the rate of convergence in the Gauss-Seidel method," Journal of Computational and Applied Mathematics, vol. 164, no. 1, pp. 587-600, 2004.

[19] J. Kennedy, Encyclopedia of Machine Learning [M], Springer, New York, USA, 2011, 760-766. 


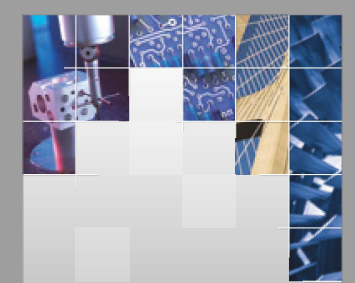

\section{Enfincering}
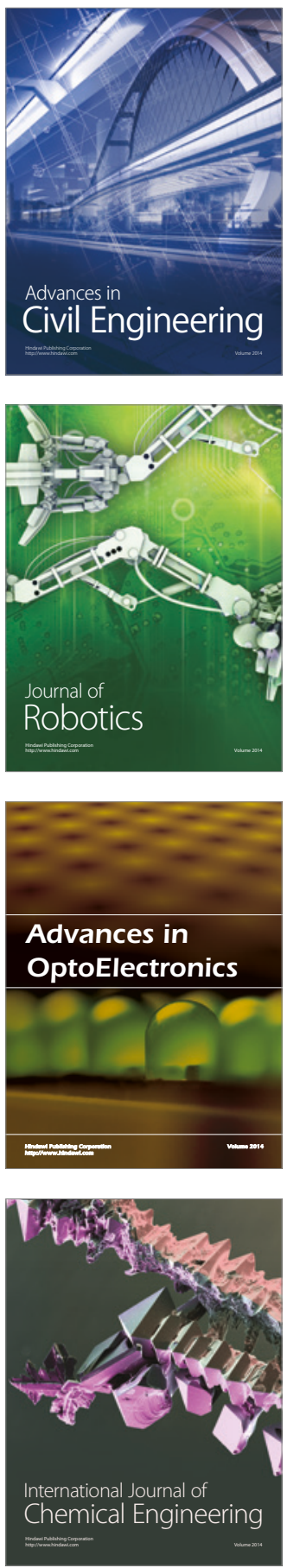

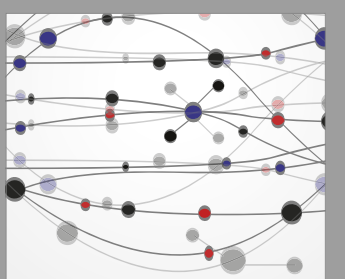

The Scientific World Journal

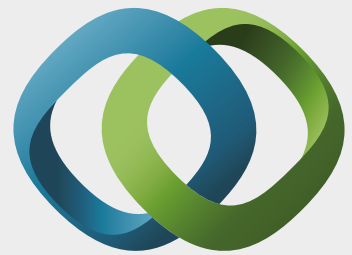

\section{Hindawi}

Submit your manuscripts at

https://www.hindawi.com
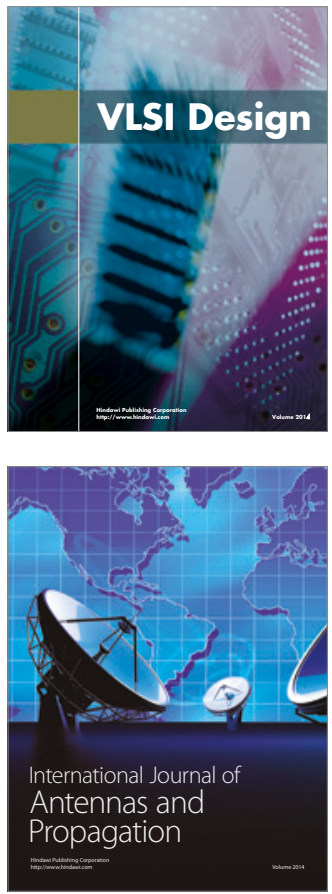

\section{Rotating}

Machinery
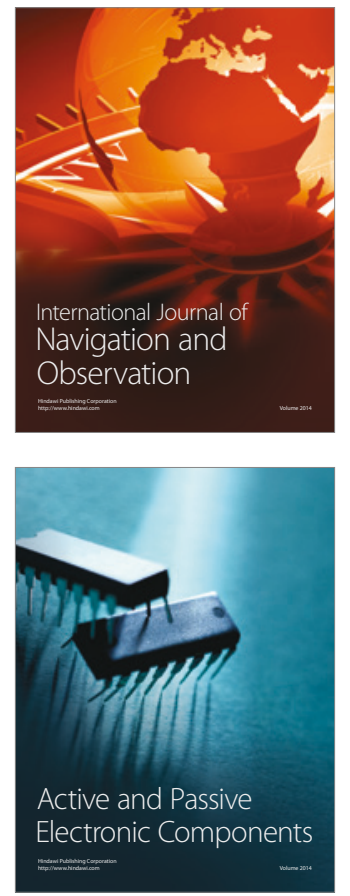
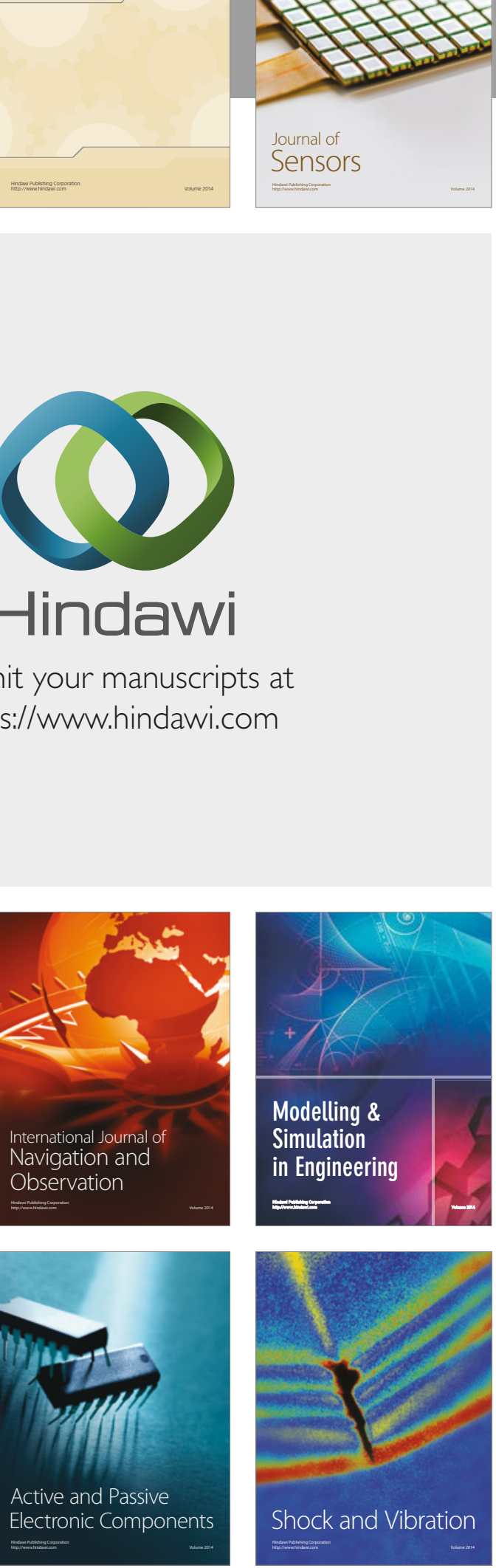
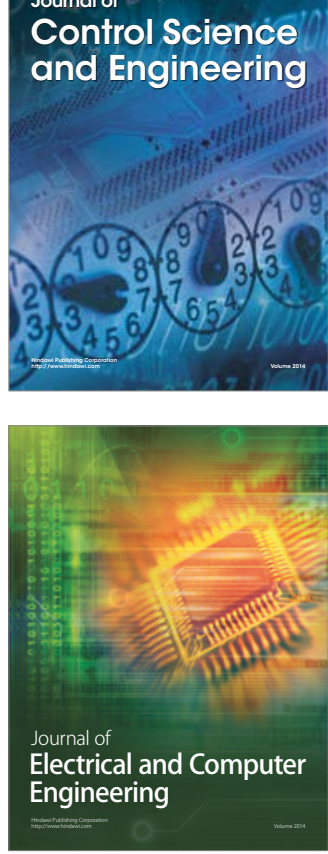

Distributed

Journal of

Control Science

and Engineering
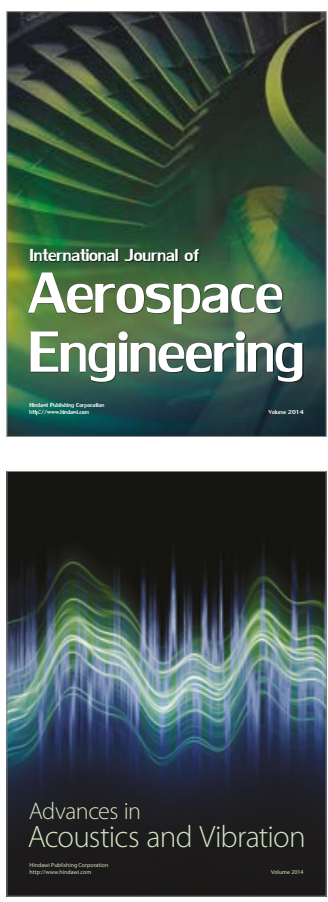

Sensor Networks 